\title{
Smart cleaning of cultural heritage: a new challenge for soft nanoscience
}

\author{
Michele Baglioni, Rodorico Giorgi, Debora Berti and Piero Baglioni $\dagger^{*}$
}

Received 22nd July 2011, Accepted 22nd September 2011

DOI: $10.1039 / \mathrm{c} 1 \mathrm{nr} 10911 \mathrm{a}$

\begin{abstract}
The search for innovative, smart and performing cleaning agents is one of the main issues of modern conservation science. Nanosciences do not only provide solutions to this scientific field in terms of new materials but also change radically the approach to problems and challenges. In this feature article we review the most innovative nanostructured systems developed in the last decade for the cleaning of artworks together with some noteworthy case studies. Micelles, microemulsions, thickened complex fluids, and responsive gels that constitute the new "cleaning palette" for modern conservators are here presented and critically analyzed. The development of these smart nanostructured systems requires the comprehension of their behavior and interactions with other materials down to the nanoscale. In the last section of this manuscript we report on the most recent results from a study about the mechanism of polymer removal from porous artifacts using nanofluids, such as micelles or microemulsions. The rules of classical detergency do not fully address the polymer removal mechanism and a schematic model of the process is proposed.
\end{abstract}

\section{Introduction: cleaning of artworks}

The removal of undesired material from the surface of an artifact is one of the most important and delicate operations in the

Chemistry Department and CSGI, University of Florence, via della Lastruccia 3, Sesto Fiorentino, Italy.E-mail: baglioni@csgi.unifi.it; Fax: +39055 457 3036; Tel: +39055 4573033

$\uparrow$ No kinship exists among the authors. conservation of cultural heritage. The generic term "undesired material" stands for a wide group of substances, which can be found on artworks due to natural ageing (e.g. grime and dirt), extraordinary events (e.g. mud and soil from floods or other catastrophic events), wrong restoration interventions, or even due to the deliberate will of the artist, as for the case of varnishes that degrade and darken over time and must be periodically replaced. Despite the apparent simplicity, cleaning is a challenging issue both for conservators and scientists. The former

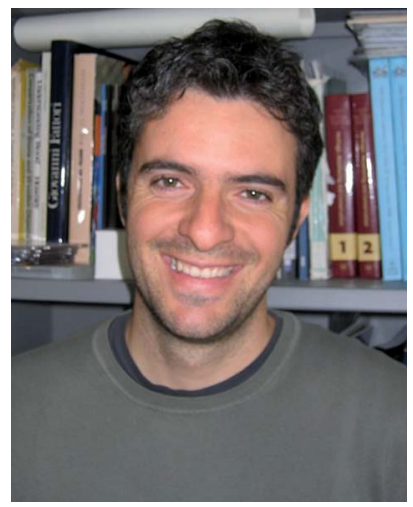

Michele Baglioni
Michele Baglioni has obtained PhD in Science for Cultural Heritage Conservation at the University of Florence and BS in Technology for the Conservation of Cultural Heritage in 2007. He is currently working as a postdoctoral fellow at CSGI in the development and characterization of soft matter systems for conservation. His research mainly deals with the study of nanostructured media for the cleaning of wall paintings both from a physical-chemical and an applicative standpoint.

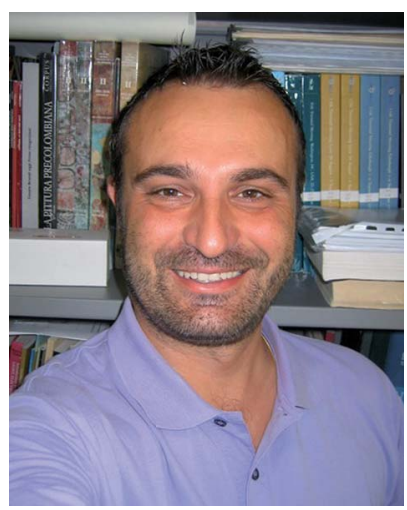

Rodorico Giorgi
Rodorico Giorgi has obtained PhD in Science for Cultural Heritage Conservation at the University of Florence and BS in Chemistry. He is currently a research fellow at the Department of Chemistry and CSGI. Giorgi's background is in physical chemistry of colloid and interfaces. Main research interests are in physico-chemical characterization of materials, investigation of degradation processes and development of methodologies for the conservation of different works of art materials, such as wall and canvas paintings, stone, paper, and archaeological wood. Giorgi is author of more than 60 publications in the field of nanotechnology and soft matter applied to the conservation of Cultural Heritage. 
have to evaluate the option of removing some material from an artwork on the basis of historic, ethical, esthetical and sometimes philosophical considerations, while the latter should provide an effective and safe method to perform this operation. From a chemical point of view, cleaning consists in finding the way to remove (i.e. to solubilize, swell, hydrolyze) specific materials without affecting the others. In other words, this operation must be controlled and selective. In the cleaning of artworks, conservators deal with almost all kinds of materials (from siccative oils to inorganic salts concretions, from black crusts to synthetic organic polymers, etc.), but in each case the quest for better performing and more selective cleaning tools is constant.

Modern conservation science is a relatively young discipline, but in the few years of its existence the search for innovative cleaning tools has been one of its main goals. The availability of a great choice of pure organic solvents at first opened up new perspectives for cleaning operations. Nowadays, most organic solvents are not the preferred option, in view of their toxicity and the disposal and recycling concerns.

In the past centuries, artists and early conservators experimented almost every kind of possibility to find effective cleaning agents on a trial-and-error basis. Traditional cleaning methods included the use of wine, vinegar, lemon juice, potash solutions, sliced potatoes, onion or garlic; also physiological fluids were widely used, such as urine, bile fluids, blood or saliva. ${ }^{1}$ These formulations already contained almost all the classes of components and active principles used in modern cleaning media, even though the contribution of each ingredient to the final outcome of the process was essentially not understood. Wine, which basically can be thought of as a mixture of water and ethanol, can be considered the precursor of modern solvent blends; vinegar, with its content in acetic acid, was an effective powerful cleaning agent able to dissolve materials otherwise insoluble at higher $\mathrm{pH}$. Saliva is maybe one of the most complete and complex traditional cleaning systems ever employed: nowadays a non-biodegradable synthetic version of this physiological fluid is still used. ${ }^{\mathbf{1 , 2}}$

A class of compounds present in the above mentioned physiological fluids is represented by surface-active agents. Surfactants are important components of bile fluids, saliva and other natural products. Soaps derived from vegetal oils or animal fats have been also known since ancient times, but the huge diffusion of these substances boosted by cosmetic and detergent chemical companies during the last century, combined with the extensive experimental and theoretical efforts aimed at the comprehension of the connection between phase behavior and microstructure, opened up unexpected fundamental and applicative horizons in different areas.

Binary or ternary systems composed of water, surfactants and other additives belong to the realm of soft matter and have characteristic length scales in the nanometric domain, which largely determine their interaction with complex micro-structured composite surfaces, such as pictorial layers. In this respect, their characterization, both from a structural and a dynamical point of view, requires the know-how of colloids and nanoscience. Moreover, if the efficacy as cleaning agent is to be predicted, assessed or improved, the interaction mechanism with the material to be removed from the work of art needs to be understood down to the nanoscale.

For the above reasons it is clear that the synergy among nanosciences, surface science and conservation science has been and will be the key for the development of some of the most powerful yet mild and environmentally friendly cleaning tools.

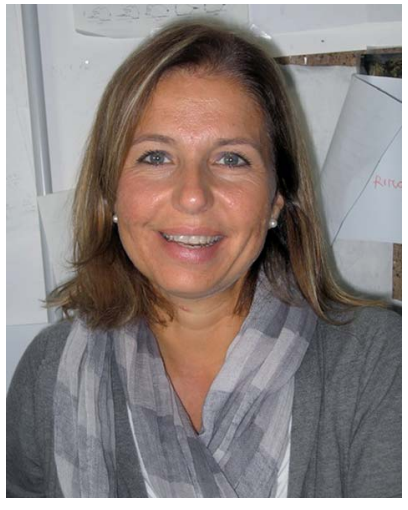

Debora Berti
Debora Berti has obtained Laurea Degree in Chemistry and the PhD in Physical Chemistry from the University of Florence. She is avisiting scientist at the ETH (Zurich) and post-doctoral fellow of CSGI (Italian Center for Colloids Nanoscience). At the University of Florence, she teaches Physical Chemistry of Nanosystems and Colloid Chemistry. Her background is in physical chemistry of selfassembly of bio-inspired and biorelevant functional amphiphiles (nucleolipids). Her research topics include: nanostructured fluids for the Conservation of Cultural Heritage; hybrid nanoparticlellipid assemblies for stimuli responsive drug delivery; and interaction of nanostructured assemblies with model membranes. She is author of more than 70 research papers. She has been member of the European Colloid and Interface Society, American Chemical Society, Italian Chemical Society, European Neutron Scattering Association, Italian Society for Neutron Scattering, IACIS. She is the President Elect of the European Colloid and Interface Society.

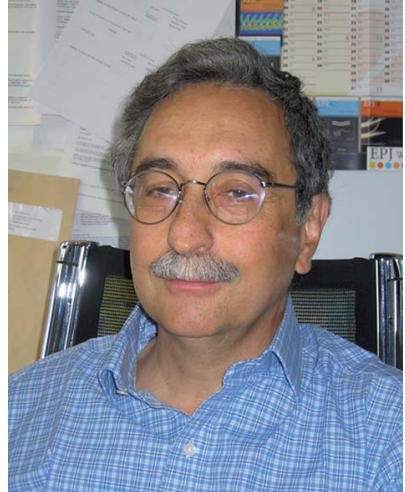

Piero Baglioni
PieroBaglioni is a Full Professor of Physical Chemistry at the Department of Chemistry and CSGI of the University of Florence. He is the author of more than 300 publications in the field of colloids and interfaces and a pioneer in the application of soft matter to the conservation of Cultural Heritage. He has produced several innovative methods for restoration of works of art, which are applied worldwide. 
This paper provides (i) an overview of the work done throughout the years about this particular subject and (ii) some considerations of the authors about the current state of the art and the most crucial issues that should be addressed in the future. Finally, some of the last innovative results concerning the study of cleaning processes will be presented and discussed.

\section{Nanofluids as cleaning agents}

\subsection{Detergency properties of surfactant solutions}

Binary water/soap systems can detach grime and fatty materials from surfaces and disperse or dissolve them in the aqueous medium. Despite the seemingly banality of this statement, detergency is a very complex phenomenon that rarely involves solubilization in its commonly accepted meaning.

The formation of surfactant aggregates above a critical concentration threshold can be thought of as a pseudo-phase separation that creates nano-sized dynamic compartments, with different dielectric properties with respect to the bulk solvents, ${ }^{3,4}$ that break and recombine on the milliseconds (ms) time scale.

The diffusion coefficients of micelles are of the order of $10^{-6} \mathrm{~cm}^{2} \mathrm{~s}^{-1}$ (for a micelle of $3 \mathrm{~nm}$ radius in water at room temperature) and the apolar/water interface is constituted by the polar heads region, which typically possesses dielectric constants similar to neat short-chain alcohols and in some cases have a net charge. ${ }^{5}$ Therefore the properties of the fluid are not isotropic at the nanoscale and a number of different environments is present in a macroscopically monophasic system.

Direct micelles are able to include in one of these compartments (i.e. in the hydrophobic core, the interfacial region or the peripheral hydrophilic region) otherwise insoluble substances with the result of dispersing them in aqueous solution. In the course of this process, bulk interfacial phenomena are equally important and involve adsorption of surfactant molecules at the interface of water and the apolar deposits.

The most important mechanisms for the removal of oily soils promoted by binary micellar solutions are roll-up, emulsification, and solubilization. ${ }^{4}$ In the latter process, the oily soil is often dispersed into a microemulsion that forms in situ. Studies about detergency mechanisms demonstrated that the best cleaning performances with surfactant-based aqueous solutions are indeed obtained when the interaction with oily soils leads to the formation of microemulsions. ${ }^{6-13}$

\subsection{Detergency properties of microemulsions}

Microemulsions (see Fig. 1) represent other important amphiphile-based colloidal systems, which have attracted many researchers because of the wide number of possible applications, from drug delivery to food science, to biotechnology and to template-directed synthesis of both inorganic and organic nanoparticles. ${ }^{\text {,14-16 }}$ Besides these traditional applications, these systems also represent a significant step forward in conservation science, with regard to the development of new cleaning tools.

A rigorous definition of a microemulsion and a clear distinction from simple or complex micellar aggregates is not an "easy task", and sometimes terms such as swollen micelles are used. We report here two concise yet rigorous definitions provided by some of the founding fathers of the field. "A microemulsion is defined as

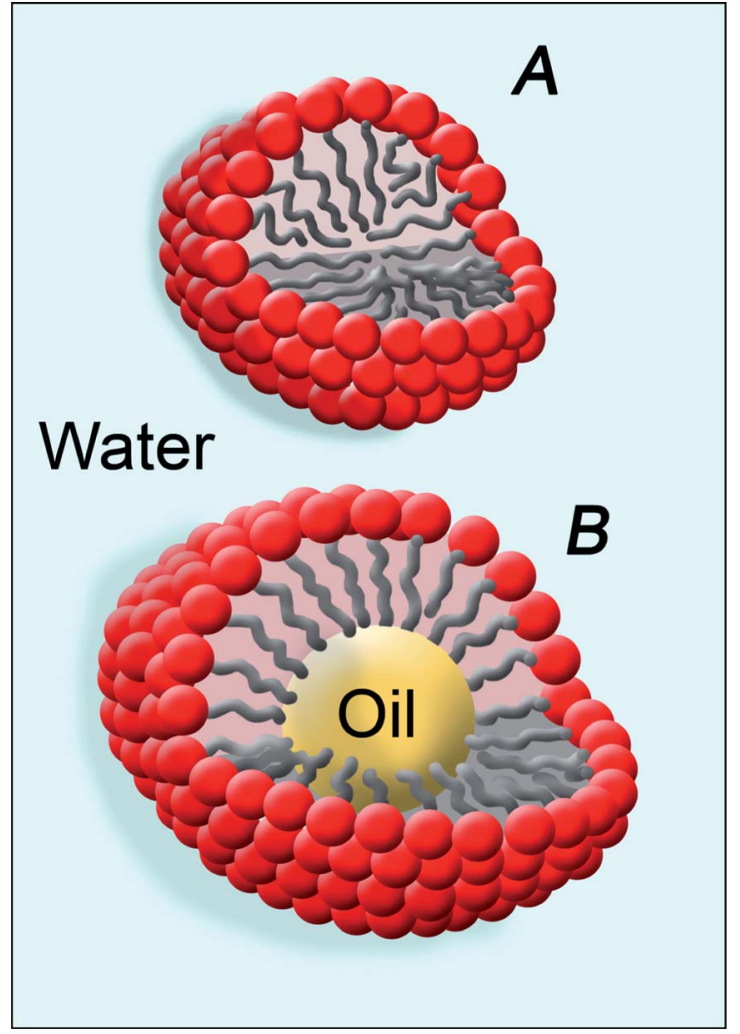

Fig. 1 Schematic representation of micelles (A) and oil-in-water microemulsions (B). These nanostructured systems are the most performing cleaning tools available to remove polymer coatings from artifacts.

a system of water, oil and amphiphile, which is a single optically isotropic and thermodynamically stable liquid solution"; ${ }^{17}$ and "We $[\ldots]$ use the term microemulsion to mean a thermodynamically stable dispersion of large oil and surfactant or water and surfactant aggregates". ${ }^{18}$

The thermodynamic stability of microemulsions derives from the energetic balance between the entropic dispersion in water of a given oil volume fraction in numerous nanodroplets, the enthalpic cost of interface creation (compensated by the lowering of the surface tension at the oil/water interface provided by the surfactant film) and an elastic term which accounts for the cost to bend the surfactant film away from its spontaneous curvature. In other terms the dispersing "power" of a binary amphiphilic aqueous system towards oil is dictated by thermodynamic parameters, ultimately connected to molecular properties. ${ }^{18,19}$

Microemulsions also possess detergency properties and can be used for cleaning purposes. The detergent properties of microemulsions have been less studied than those of common surfactant solutions, in view of the lower applicative impact as direct cleaning media in everyday life, ${ }^{20}$ but some examples exist about this subject (e.g. textile cleaning, hard surface cleaning, vehicle cleaning, dry cleaning). ${ }^{21}$

The detergent power of a generic "oil-dispersed-in-water" system $(\mathrm{o} / \mathrm{w})$ is given by the combination of more factors: the detergent properties of micellar solutions, the solving power of organic solvents, and the extension of the interfacial area of the system itself. For some applications (i.e. when detergent systems 
should only provide a surfactant reservoir) microemulsions and micellar solutions are equally suitable. However, when high solubilization power is needed, microemulsions are undoubtedly the most performing systems. ${ }^{13}$ Detergency and soil removal applications benefit from ultra-low interfacial tension that can be achieved with microemulsions ${ }^{16}$ and it has been demonstrated that on textiles these systems provide better cleaning results than surfactant solutions, even at low temperature and with minimum need for mechanical energy. ${ }^{22-24}$

\subsection{Microemulsions for the cleaning of artworks - the first tests}

The introduction of amphiphile-based nanostructured systems as cleaning tools for artworks is relatively recent. The first application of microemulsions for the conservation of cultural heritage was performed by E. Ferroni and P. Baglioni at the end of the ' $80 \mathrm{~s}$, during the restoration of the Renaissance paintings in the Brancacci chapel in Florence by Masaccio, Masolino, and Lippi. ${ }^{25,26}$ The presence of a multitude of wax spots due to the extinction of votive candles kept close to the mural paintings was detected through an investigation with UV light. The removal of this hydrophobic material from the hydrophilic fresco painting required an aqueous-based system that was able to dissolve the apolar waxy material without re-dissolving it in the porous matrix of the wall. A paper from Pierre Gilles De Gennes and Christiane Taupin about interfacial curvature in microemulsions ${ }^{27}$ inspired the first o/w microemulsion system specifically tailored for the cleaning of works of art surfaces. A microemulsion, composed of dodecane nanodroplets stabilized in water by ammonium dodecylsulfate and 1-pentanol (1-PeOH), was used to clean the Brancacci chapel's paintings. The wax was dissolved by dodecane and confined into the microemulsion droplets, avoiding its spreading into the wall, which would have occurred with a pure solvent. Excellent results were obtained by means of this innovative approach, reducing the side effects of a traditional cleaning procedure. ${ }^{26}$ The successful application of this microemulsion showed the potentiality of these aqueousbased methods as cleaning tools for wall paintings.

\subsection{Polymer removal from wall paintings}

Besides the traditional consolidants (i.e. animal glues, polysaccharides, plants extracts, milk, egg white, siccative oils, natural resins and $\mathrm{wax}^{28}$ ) synthetic polymers have been widely applied as consolidating agents, but their use represents a severe threat for several different classes of artifacts and in particular for wall paintings. The use of synthetic polymers in conservation probably reached its peak of diffusion during the second half of the $1960 \mathrm{~s} .{ }^{28,29}$ Contrary to the expectations, the multitude of wall paintings treated with polymers during the second half of $20^{\text {th }}$ century showed over time very poor conservation states, and, in some cases, accelerated degradation due to the presence of the polymeric products that should have guaranteed their durability.

The reasons for this behavior became soon evident. ${ }^{30}$ When a polymer is applied on a porous support a surface film is invariably formed even using very low polymer concentrations. The presence of a polymer film over the artifact's surface drastically changes surface permeability to gases and liquids, leading to mechanical stresses and to the degradation of the pictorial layer that is further amplified by the possible presence of salts. These are forced to crystallize below the pictorial region leading to the complete disintegration of the rendering layers.

Additional long-term effects are related to the degradation of the polymer itself. Commercial synthetic polymers have been designed and characterized for purposes different from their use in restoration, where long durability is required. As an example: an acrylic polymer is produced to last 20-30 years as a binder for paints, but if the same polymer is applied on a wall painting, this durability is obviously inadequate. As a matter of fact polymers applied on wall paintings degrade in a few decades and often become discolored and brittle, compromising the readability and the integrity of artworks. Nowadays conservators are trying to remediate years of unawareness removing the polymers applied in previous restoration interventions.

In most cases a polymer film can be removed from a porous material with an organic solvent, but this choice has a series of drawbacks. First, the action of an organic solvent is often poorly controllable. Moreover, solvents convey the dissolved polymer in the interior of the mural matrix and redeposit it deeply in the pores. In fact, most of organic solvents quickly evaporate from the wall, while polymer macromolecules, which diffuse sensibly slower, tend to remain inside the porous matrix. Finally, the toxicity of most of the solvents used in these operations represents a serious problem. Since many wall paintings have wide surfaces and are conserved in poorly ventilated environments, safety is of fundamental importance for the operators' health.

Micelles and microemulsions represent the most interesting alternatives to the use of pure organic solvents. Using these aqueous nanostructured systems, it is possible to obtain a controlled, and in certain cases selective, removal operation. They are low-toxicity cleaning tools that possess high effectiveness and permit to avoid the side effects of neat solvents, such as polymer redeposition. Another important feature is that some polymers, which, upon ageing, became insoluble or hardly soluble in solvents or solvent mixtures, are often removable by the use of microemulsions. Polymer solubilization mechanisms are not simple to investigate, especially when macromolecules are altered by ageing, and often solubilization is not the only process involved in polymer removal, as we will see in the following sections where we will review the work presented on the removal of the two main classes of polymeric consolidants, i.e. acrylic and vinyl polymers.

\subsection{Nanofluids for the removal of acrylic coatings}

Acrylic polymers are probably the most diffused synthetic compounds used in the conservation of cultural heritage. The necessity of removing these substances from the surface of previously preserved artifacts became evident at the end of 1990s already; two formulations were developed to remove this class of substances. Both systems contain xylene as the dispersed phase ${ }^{31,32}$ because of its high affinity for acrylic resins. ${ }^{33-35}$ These two nanofluids contain Tween80 (polyoxyethylene sorbitan monooleate)/1,2-propandiol and sodium dodecylsulfate (SDS)/1$\mathrm{PeOH}$ respectively as surfactant/co-surfactant couples. Both systems efficiently removed Paraloid B72 (a 70:30 ethyl methacrylate/methyl acrylate copolymer; Rohm \& Haas, USA; see 
Fig. 2) coatings both from test glass slides and mural paintings. Hereinafter, the microemulsion containing xylene, SDS and 1$\mathrm{PeOH}$ will be addressed as "XYL". The XYL system is a fourcomponent microemulsion that was developed on the basis of the classic work by Rance and Friberg. ${ }^{34}$ A complete structural characterization of this system has been recently carried out. ${ }^{36}$ The XYL system was successfully tested in the removal of acrylic coatings from the wall paintings by Spinello Aretino, in the Cappella Guasconi (San Francesco church, Arezzo, Italy). ${ }^{31}$ The addition of a commercial solvent mixture, known as "nitro diluent" $\$$ because of its ability to dissolve nitrocellulose-based paints, to the XYL system was then proposed. ${ }^{37}$ The effect of this addition was the enhancement of the effectiveness in the removal of the acrylic coatings removal. This system will be named "XYL-ND" in the following. XYL-ND is a five-component system, but since nitro diluent is a mixture of five solvents by itself, it is actually composed of nine different compounds, though some of them are present in almost negligible amounts. This system was characterized through QELS analyses and a hydrodynamic diameter of $17.8 \mathrm{~nm}$ was obtained for its droplets. ${ }^{37}$ As for the XYL system it was tested on the frescoes by Spinello Aretino and it provided excellent cleaning activity.

\subsection{Amphiphile-based systems for the removal of vinyl polymers from wall paintings}

The XYL-ND microemulsion was also tested in 2003 in the restoration of Conegliano Cathedral (Italy) on a fresco treated with poly(vinyl acetate). ${ }^{37}$ The results of preliminary tests were not satisfactory, indicating that this microemulsive system was not appropriate for vinyl polymers removal. The reason lies in the fact that $p$-xylene is not a good solvent for vinyl polymers; ${ }^{38}$ rather, more polar solvents, such as propylene carbonate (PC), which has good solubility in water $(\sim 20 \% \mathrm{w} / \mathrm{w})$, can be used for this purpose. Therefore, for the removal of vinyl polymers was proposed a SDS/1-PeOH and PC micellar solution. ${ }^{37}$ Many different systems containing $\mathrm{PC}$ were investigated (neat $\mathrm{PC}, \mathrm{PC} /$ 1-PeOH, PC/SDS/water, and PC/SDS/water/1-PeOH) and tested on the Conegliano wall paintings, but only the quaternary system succeeded in the complete removal of the vinyl coating. This system is hereinafter named "PC22" because of the $22 \%$ content
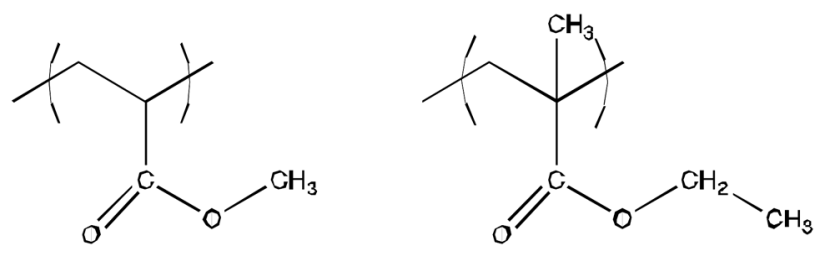

Fig. 2 Chemical structure of Paraloid B72, one of the most common acrylic polymers used in conservation of cultural heritage. Paraloid B72 is a random copolymer of methyl acrylate (left), 30\%, and ethyl methacrylate (right), $70 \%$.

$\$$ Nitro diluent is a commercial solvent mixture composed $(\% \mathrm{w} / \mathrm{w})$ of toluene, $62 \%$; butyl acetate, $15 \%$; ethyl acetate, $15 \%$; butanol, $6 \%$; Cellosolve acetate, $2 \%$. (w/w) in propylene carbonate. PC22 is a peculiar four-component system. It has been defined for years as a micellar solution. Strictly speaking, it is something different from a simple dispersion of surfactant micelles in water. QELS measurements ${ }^{37}$ did not show significant changes in the SDS/1-PeOH micelle structure after the addition of $\mathrm{PC}$, even at high concentrations. This is probably due to the fact that PC contained in the micellar phase resides at the aggregates surface, rather than in their hydrophobic core. The PC22 system was further investigated by Palazzo et al..$^{39}$ and Colafemmina et al., ${ }^{40}$ and more information about its partition and localization in the micellar structure of the system was obtained. It has been found that PC is mainly solubilized in the water phase, but a molar fraction of about $40 \%$ is contained in the micelles, where it acts as a co-surfactant and increases the surface area of the aggregates.

Recently, a system whose composition was very close to the PC22 system was successfully used in the removal of acrylic polymers (i.e. Paraloid B72) from mural paintings in the church of Santa Maria della Scala in Siena. ${ }^{41}$ These tests showed that a system that was designed to intervene on vinyl polymers could be used also to remove acrylic resins, extending the cleaning "palette" in the hands of restorers.

\subsection{The wall paintings of Mayapan and the $E A P C$ system}

A cooperation project with the Instituto National de Antropologia e Historia (INAH) of Mexico City was undertaken some years ago by some of the authors of this paper with the aim of finding novel formulations for the conservation of archeological sites in the Pre-Colombian area. Nowadays, Mexican conservators have to face the decaying of wonderful Maya and Nahua mural paintings discovered in Mexico. As an example we report on the Mayapan paintings (Yucatan, Mexico; see Fig. 3), which are literally disintegrating as a consequence of inappropriate conservative treatments. A coating of Mowilith DM5 (a $65: 35$ vinyl acetate/ $n$-butyl acrylate copolymer, Hoechst, Germany; see Fig. 4) was applied on the paintings at the end of the 1990s, with both a protective and consolidating intent. However, after less than ten years conservators of the site had understood that

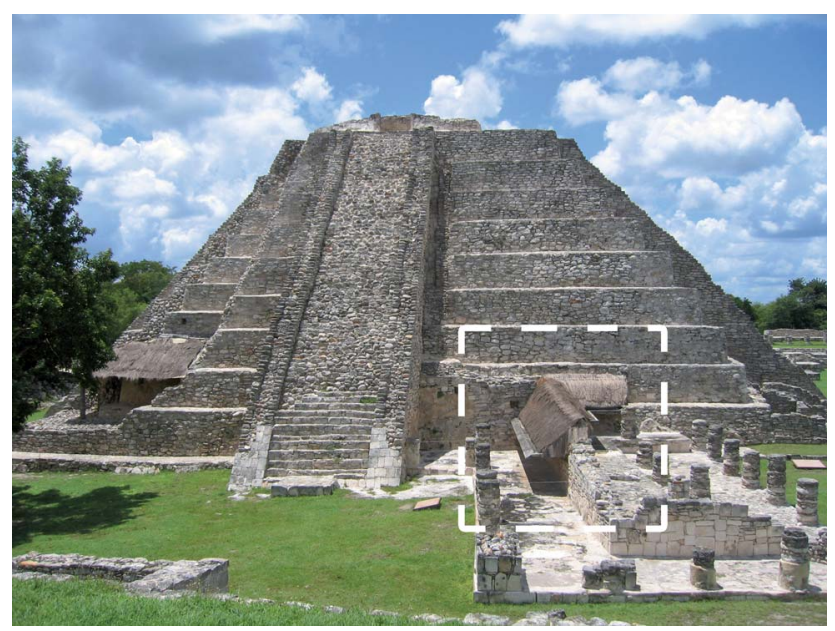

Fig. 3 View of the main building in Mayapan, Mexico. The wall painting "de los discos solares" location is indicated by the dashed line. 
<smiles>CCC(CC)OC(C)=O</smiles><smiles>CCCCOC(=O)C(CC(C)(C)C)C(C)C</smiles>

Fig. 4 Chemical structure of Mowilith DM5, a vinyl/acrylic copolymer widely used in several kinds of artifacts. In Mexico it has been used as a fixative and protective on archeological wall paintings, with devastating effects. Chemically, it is a random copolymer of vinyl acetate (left), $65 \%$, and $n$-butyl acrylate (right), $35 \%$.

Mowilith removal was the top priority for the integrity of the paintings. In 2008 they proposed us the use of ethyl acetate (EA), as they found it to be a good solvent for the vinyl/acrylic copolymer present on the paintings. The chemical nature of this copolymer, which is formed by vinyl and acrylic monomers, prompted the usage of a PC22 modified system, because of its effectiveness towards both classes of resins. Therefore ethyl acetate was added to a mixture of water, SDS, 1-PeOH and PC, in order to create a cleaning tool to intervene in Mayapan (see Fig. 5). This system was named "EAPC". ${ }^{36,42,43}$

The nature of EAPC is even more complicated than the PC22 system by the presence of ethyl acetate, which is also a partially water-soluble solvent $(\sim 8 \% \mathrm{w} / \mathrm{w})$. As shown by a detailed structural characterization ${ }^{36}$ this system differs from classical microemulsions due to the presence of two components (propylene carbonate and ethyl acetate) that are partly soluble in both the dispersed and continuous phase, making the interface of this system similar to that of a swollen micelle. With this feature in mind, to simplify the discussion we will refer to the EAPC and XYL system as microemulsions. The EAPC system was found to be very effective in the removal of several kinds of polymers. The mechanism of interaction between the EAPC system and the polymer coating during the cleaning process has been the subject of a detailed study ${ }^{42,43}$ and in the last section of this paper some recent results are reported and discussed. EAPC is one of the most effective systems so far developed for the removal of polymers from artworks.

Its performances were successfully tested in the removal of vinyl/acrylic copolymers from the paintings of Mayapan (see Fig. 6 and 7), in the removal of acrylic coatings from the
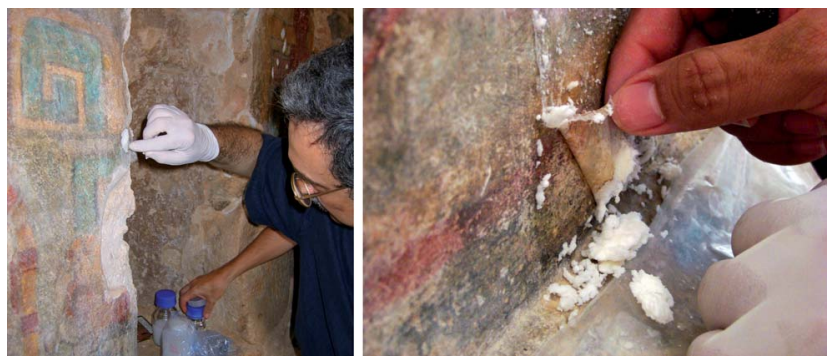

Fig. 5 The picture on the left illustrates the application procedure of the EAPC system. A cellulose poultice impregnated with EAPC is applied over the painting surface, previously protected with a Japanese paper tissue. On the right, the removal of the poultice after the cleaning procedure is shown. paintings of Cholula (Mexico), and in the removal of siliconebased resins from the mural decorations of the Annunciation Grotto adored as the Holy Mary's house in Nazareth, Israel.

\subsection{Nonionic surfactants}

All the systems described are characterized by the presence of SDS, one of the most studied and well known anionic surfactants, which possesses a good emulsifying power and excellent detergency properties. Recently, our attention was addressed to nonionic surfactants because of their very low CMC, which permits to reduce the amount of the nonvolatile fraction in cleaning systems. Alkyl polyglycosides (APGs) belong to

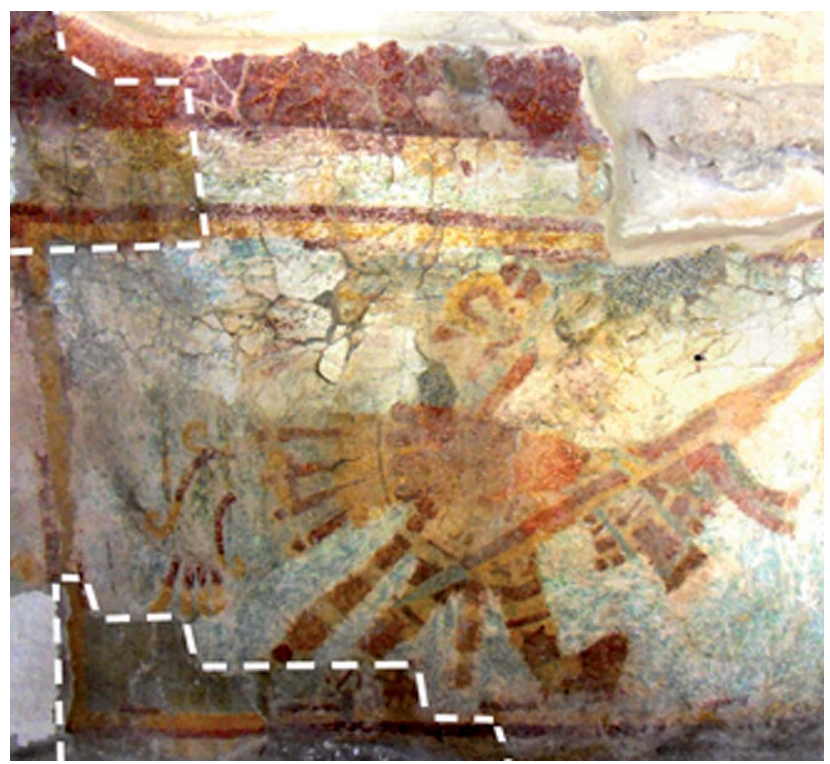

Fig. 6 A particular of the wall paintings "de los discos solares" in Mayapan (Mexico), after the removal of the polymer coating by using the EAPC system. The dashed boxes highlight small areas that were not cleaned and left untreated as a reference.
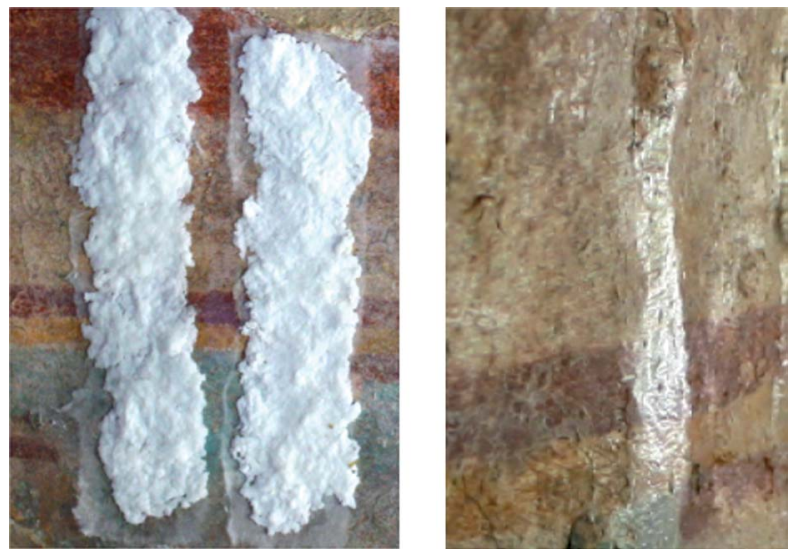

Fig. 7 On the left, application of EAPC and XYL systems for test experiments on the paintings "de los discos solares" in Mayapan, Mexico. On the right, the same area is observed after the removal of polymer coating. Under glazing light, the disappearance after treatment of the shining effect due to the coating can be appreciated. The presence of the polymer is evident in narrow strip that was left untreated. 
a relatively novel class of "green" surfactants that possess excellent detergency properties and biodegradability ${ }^{44}$ and they are synthesized from renewable raw materials. ${ }^{45,46}$ Because of their advantages from an economical and environmental point of view, these compounds are interesting for a great number of applications, including cleaning of artworks. An o/w microemulsion containing water, xylene and a mixture of APGs was recently developed, which was tested in the removal of Paraloid B72 from the wall paintings in Santa Maria della Scala (Siena). ${ }^{47,48}$

Nonionic surfactants have also the advantage of being almost insensitive to ionic strength. Microemulsion formed from these surfactants can be therefore loaded with salts, and in particular with ammonium carbonate, that is very efficient in swelling grime and very helpful in the removal of sulfates (one of the most common and harmful salt promoting frescoes degradation). A microemulsion constituted by Triton X-100, xylene, water and ammonium carbonate was formulated by Carretti et al. in 2007.47 This system combined the detergent power of a microemulsion and the desulfation effect due to the presence of ammonium carbonate, which was originally used in the first step of the "Ferroni-Dini method". ${ }^{49-51}$ The ammonium carbonate present in the microemulsion reacted with calcium sulfate to give a powdery calcium carbonate and the soluble ammonium sulfate that dissolves in the water phase of the microemulsion or can be easily washed away, allowing the removal of materials that could not be removed by using conventional cleaning methods.

\section{Cleaning: solvents' thickeners for the conservation of cultural heritage}

\subsection{The importance of controlling the cleaning action}

The cleaning action performed with free organic solvents is too scarcely controlled and poorly selective. Moreover, very often organic solvents are too volatile or their retention on the artwork is too high for an effective and safe application. Finally, the toxicity of the majority of these compounds, as previously stressed, is not negligible in this context. To overcome these problems pastes and poultices constituted by organic solvents and waxes were developed already in the first half of the last century. ${ }^{52}$ Wax reduces solvent migration and decreases its evaporation rate. These systems presented several limitations due to the difficult preparation and the high amount of residues that remained on the treated surface. The natural evolution of waxbased poultices was represented by gelled systems. Gels and solvents' thickeners composed of organic solvents and modified celluloses, poly(acrylic acid), or other high molecular weight compounds were readily adopted by conservators due to their high versatility in cleaning operations and to the high solvent/ gelator ratio that can be reached in these systems. ${ }^{53}$ The main advantages coming from the use of gelled systems in cleaning operations are related to these two facts: (i) the cleaning processes are more controlled and effective and (ii) safety for the operators is increased. This is mainly due to the increase of the retention time of the solvent, and at the same time to the decrease of capillary penetration inside the artifact. Moreover gelled formulations enhance the solubilization power of the solvent itself because the decrease in the evaporation rate leads to the creation of a microenvironment where liquid solvent and solvent vapors realize a particularly effective cleaning action. ${ }^{54}$ Finally, gels or thickeners limit solvent evaporation and thus the exposition of the operators to toxic vapors is sensibly reduced.

\subsection{Gelled systems for the cleaning of artworks}

The increase of "solvent" viscosity produced by thickeners, and in particular by poly(acrylic acid) as a gelator, ${ }^{1,54-56}$ offered new cleaning systems to conservators and conservation scientists. ${ }^{54}$ At the end of the 1980s, R. C. Wolbers proposed the use of new aqueous thickened cleaning systems. ${ }^{55,57,58}$ According to the "active principles" contained in the formulation of these systems, they are classifiable in three categories: gels $\S$ with enzymes, resin soaps, and solvent gels. Besides their main components (respectively enzymes, terpenoids, surfactants and organic solvents), the systems proposed by Wolbers may also contain salts, $\mathrm{pH}$ buffers, chelants, acidic or basic compounds, surfactants, and, of course, water. Later Wolbers reported ${ }^{1}$ on a ternary system composed of water, Triton X-100 and xylene similar to the one described in Section 2.8. This system can form emulsions, o/w microemulsions or w/o microemulsions depending on the relative amounts of the three components. The systems used by Wolbers are actually emulsions rather than microemulsions, thickened by high molecular weight modified celluloses, in order to obtain gel pastes that could be brushed on the artwork.

Thickened systems had a strong impact on conservation of cultural heritage and on conservation science. As a matter of fact, they definitely possess some advantages and constitute some of the most popular cleaning systems among the conservator community. Especially solvent gels, which often substitute free organic solvents partly overcoming their side effects, seem to represent a class of particularly interesting cleaning systems. Notwithstanding their improvements over common organic solvents, they present some critical drawbacks. All solvent gels nowadays used in restoration are "physical" gels and this means that their structural network is based on weak secondary interactions (e.g. dipole-dipole interactions, hydrogen bonds, van der Waals forces). These systems can be very hardly removed from an artwork porous surface after their cleaning action is completed and often conservators are forced to use neat organic solvents for the clearance of the same gels that were applied to avoid the use of free liquids. This is an understandably debated issue that represents a key aspect in the gel controversy. The development of innovative gels was mainly undertaken by us with the aim of finding new solutions to the possible residues left by thickened solutions and to the control of the cleaning procedure.

\subsection{Innovative gels for the conservation of cultural heritage}

Responsive gels have been formulated for a variety of purposes, ${ }^{59-62}$ but their application to conservation of cultural heritage was not explored although their manipulation and removal are easier than traditional "physical" solvent gels.

$\S$ We are using the term gel even for the systems that are not truly gels according to their rheological behavior, but they are rather simply viscous systems. The use of this term is improper but justified by its use by conservators. 
Among the gels proposed for the application in Cultural Heritage conservation, some respond to a physical-chemical switch (a change of their $\mathrm{pH}$, for example) and can be converted to free flowing liquids to ease their removal from the cleaned surface. ${ }^{63,64}$ More recently the development of "chemical" (i.e. the structural network is based on covalent bonds) gels, based on poly(acrylamide) polymeric network, functionalized with magnetic nanoparticles led to a new kind of cleaning tools responsive to external magnetic fields. These systems can be removed from the cleaned surface simply by using a permanent magnet, which minimizes the contact between the operator and the artwork, making the cleaning safer and fully controlled. ${ }^{65,66}$

A new class of high viscosity polymeric dispersions (HVPDs) for the cleaning of artwork has been also recently presented. ${ }^{67-69}$ In analogy to the previously discussed Wolbers' gels, these systems cannot be considered as true gels but due to this peculiar behavior they are extremely promising tools for conservation of cultural heritage. The gel networks are based on the cross-linking reaction between poly(vinyl alcohol) and borate (PVA-borate systems). They belong to the class of hydrogels and can be loaded with polar solvents, water/solvent mixtures and/or salts. After the cleaning HVPDs can be easily peeled off the surface without the use of free solvents or invasive mechanical action, as is needed for traditional solvent gels.

Besides their remarkable features, gels are used as a solvent vehicle for cleaning operations, and, depending on their nature, they can be loaded with organic solvents or aqueous systems. Recent studies proposed the combined use of gels and microemulsions or micelles ${ }^{65,66,70,71}$ to create a new class of nanostructured versatile cleaning media that can be safely applied both on easel and wall paintings enhancing the cleaning capacity and minimizing the solvent release in the works of art and in the environment. Some other systems (developed for different purposes) exist, which present good potentiality for a future application in cleaning of artworks. ${ }^{72-75}$

Cellulose based supports, like wood or canvas, are highly sensitive to water and traditional aqueous systems should not be applied on these supports because they can give rise to dangerous mechanical stresses. Poly(acrylamide) chemical gels loaded with the EAPC nanostructured system were successfully employed in the removal of aged adhesives from the back of relined canvas during a study carried on in collaboration with C2MRF-Louvre Museum (Paris, France). ${ }^{71}$ These gels are highly solvent's retentive allowing to minimize the water release during their application and making the cleaning safer for the artworks. Moreover, the release of detergent aqueous systems can be controlled by modulating gels properties during the chemical synthesis (i.e. polymer molecular weight, cross linking, mesh size, etc.). The combination of gels and microemulsions probably represents the most advanced cleaning tools for conservation science produced so far.

\section{From the conservation workshop to the test tubes: some insight into the cleaning mechanism}

\subsection{Polymer removal using nanofluids: an unexplored field}

The landmark studies about detergency are mainly focused on surfactant solutions able to disperse relatively low-molecular- weight compounds, such as fatty acids, triglycerides, or in general oily soils. Referring to the tests performed on the Brancacci paintings, one can confidently state that in that case the wax removal process basically followed the rules of classical detergency as addressed in the above mentioned studies. The presence of a solvent (i.e. dodecane) enhanced the solubilization of the hydrophobic material, dispersed into the micelles.

Unfortunately this model cannot be applied to the removal of high molecular weight compounds as polymers from wall paintings. Although the effectiveness of nanostructured fluids was demonstrated in the field, the mechanisms that rule the cleaning process are not yet fully understood.

The interaction of polymers with micelles or microemulsions has been widely studied in the past, but these studies usually refer to soluble or partially soluble macromolecules as block copolymers. ${ }^{76-80}$ Conversely, very few studies have addressed the action of $\mathrm{o} / \mathrm{w}$ microemulsions on hydrophobic polymeric coatings.

The very promising results obtained with their application on wall paintings prompted us to investigate more into details the guiding principles to design and predict the performances of nanostructured fluids. ${ }^{42,43}$ The following paragraphs report the most recent results on this subject.

Our attention was focused on EAPC and XYL, which represent two excellent amphiphile-based cleaning systems. The former is the most recent nanostructured cleaning system developed by our group, while the latter is one of the first formulation used in the field.

Fig. 8 reports the SANS spectra for these formulations; without going into details, we should mention that the systems are very similar in terms of SDS and 1-pentanol concentrations; therefore their main difference, clearly visible from the SANS spectra, lies in the partition of the additional components within the "micellar compartment". A comparison between the two systems presents a definite interest in this field, in view of their different structural features at the nanoscale.

To address this point, we investigated the interaction of these two nanofluids with a film of Paraloid B72, the archetypal polymer in conservation, deposited on the bottom of a glass vial.

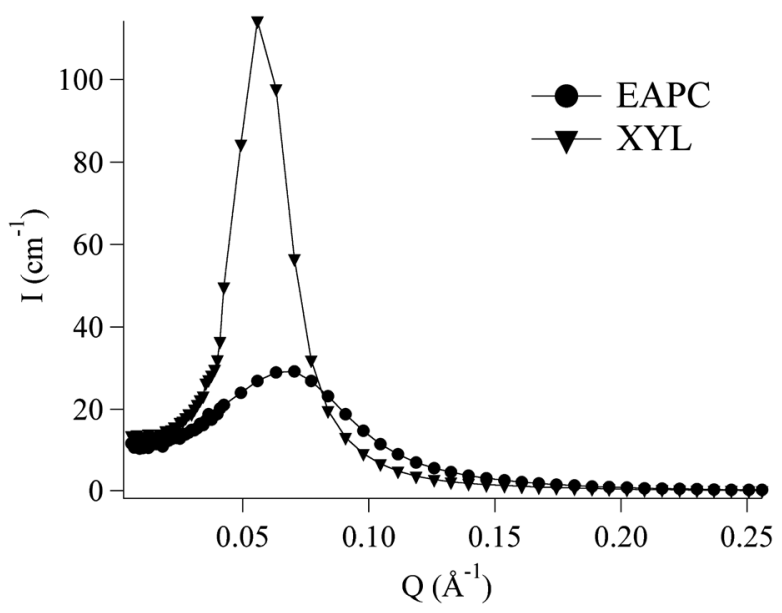

Fig. 8 SANS curves of the EAPC and XYL nanofluids. Their structure has been thoroughly analyzed in a detailed study, ${ }^{36}$ which allowed us to perform a complete structural characterization of the supramolecular aggregates. 
Some qualitative experiments were carried out, in order to have immediate visual feedback about the removal process.

The polymer is not dissolved in the aqueous nanofluid, and a phase-separated system is always the result upon the polymer/ nanofluid interaction.

From an applicative standpoint, the physical observables of interest in view of the application are the interaction rate of the process and the morphology of the polymer-rich phase at the end of the process. In some cases the polymer is simply swollen, but still stuck to the bottom of the test tube; in some other cases small droplets are visible at the bottom of the vial. The third possibility is polymer swelling, detachment and coalescence into a single drop floating onto the water phase.

To describe the kinetics of the process, the detergent systems investigated have been grouped into five "classes" for the rate of polymer removal rate (see Table 1). "Very fast" indicates a process that lasts minutes, while "very slow" refers to a process that requires some tenths of hours to reach the equilibrium. Since typical application times for detergent nanofluids on real artworks are of the order of 1-1.5 hours, "very fast" and "fast" systems are the most suitable.

In each case the process that was observed seems to have little in common with the known features of detergency. Paraloid B72 interacts with the nanofluid and swells because of solvent diffusion into the polymer film (case II diffusion ${ }^{\mathbf{8 1}, 82}$ ). This raised some questions about the exact role of surfactant aggregates: therefore the same solvent mixture as EAPC, but without SDS, was also tested (SatAq, i.e. the aqueous phase that results after separation of the excess above saturation of the organic solvents). This mixture is faster than XYL in swelling the polymer film in the test tubes, but removal tests on fresco model samples showed that both EAPC and XYL work much better than SatAq, implying that kinetics is obviously not the only important parameter. In particular, systems that promoted a spontaneous detachment of swollen polymer (like EAPC and XYL) from the bottom of the test tube have shown to be more effective than the others in real application tests.

\subsection{The same mechanism for two different systems}

Before going further with the discussion we should consider EAPC and XYL systems. The kinetics of the cleaning process is much faster for EAPC than for XYL. This difference parallels the different performances observed in Paraloid B72 removal during laboratory cleaning tests on fresco model samples.

However, for a wide range of Paraloid B72 amounts (0.1$10 \mathrm{mg}$ of polymer per $1 \mathrm{~g}$ of the detergent system) the same

Table 1 Kinetics of interaction with microemulsions for different samples

\begin{tabular}{ll}
\hline Sample & Process kinetics \\
\hline EAPC & Very fast \\
EAPC-noPeOH & Fast \\
SatAq & Moderately fast \\
SatAq $+10 \mathrm{mg} \mathrm{m}^{-1}$ SDS & Slow \\
SatAq $+40 \mathrm{mg} \mathrm{m}^{-1}$ SDS & Very slow \\
XYL & Slow \\
XYL-TW & Very slow \\
\hline
\end{tabular}

phenomenon was observed in both formulations: the formation of two liquid phases, a bulk one and, independently from the initial amount of polymer, an upper droplet which maintains the same composition (for EAPC (w/w \%): Paraloid B72, 25\%; EA, $32 \%$; 1-PeOH, 23\%; PC, 14\%; water, 6\%; for XYL (w/w \%): Paraloid B72, 25\%; xylene, 40\%; 1-PeOH, 35\%). ${ }^{43}$ It should be noted that 1-pentanol is not a good solvent for Paraloid B72; however its amount in the upper phase is considerably larger than what is expected from the composition of the detergent system.

Moreover, the amount of polymer found in the upper phase is the same for both systems and its quantity is negligible in the lower phase. After the interaction with the polymer film, microemulsion droplets decrease in size and a volume reduction of the aggregates of about $30 \%$ is observed in both systems. To summarize: after the interaction with the polymer, both EAPC and XYL are still nano-structured fluids containing microemulsion droplets, which are now smaller, due to the depletion in organic solvents. These solvents migrate from the detergent system to the polymer and promote its swelling, leading to its detachment from the surface where it was applied, which now is clean. Even if the final result is similar, the slower kinetics of the XYL system calls for a deeper investigation into the mechanism of interaction.

To address this point, we have treated separately the role that two key components, i.e. the surfactant and $\mathrm{PeOH}$, play in the removal process.

The surfactant has a very different relevance in the two systems. For the XYL microemulsion, without surfactant xylene and water would not mix, while for EAPC both PC and EA are partially water soluble. Therefore, if SDS is removed from the formulation a phase separation occurs and two phases form: a saturated aqueous solution of organic solvents (SatAq) and an excess of organic phase. This saturated aqueous phase swells Paraloid B72, but the more SDS is added to it, the slower the interaction becomes, until eventually no interaction at all occurs and the polymer film is practically unperturbed. It is clear that SDS additions to SatAq alter the partition of the solvents, shifting their distribution from the dispersing medium to the dispersed droplets: therefore EAPC, which contains the same amount of SDS as the SatAq $+40 \mathrm{mg} \mathrm{ml}^{-1}$ SDS (but a higher overall amount of organic solvents), is highly effective in polymer removal, while SatAq $+40 \mathrm{mg} \mathrm{ml}^{-1} \mathrm{SDS}$ is practically not active.

Therefore, unlike in classical detergency, the dispersed nanodroplets act as a dynamic reservoir of the solvents. The solvents are readily available to swell the polymer film: moreover the aqueous phase, at the end of the process, results in depletion of a precise solvent mixture, whose composition depends on the nature of the polymer and not on its amount.

The solvents dissolved in the water continuous phase are the first to diffuse into the polymer coating, and this could account for the fast rate of the interaction process of EAPC. After this first diffusion in the glassy polymer, a series of exchanges takes place, where solvents diffuse from water to the polymer, from the nanodroplets to water, and from the nanodroplets to the polymer. The first of these three exchanges is fast, while the other two are slower. The result of this process is a structural re-arrangement of the nanodroplets and polymer swelling, which is followed by film disruption to form small droplets that detach from 
the surface. This hypothesis can also account for the different kinetics of the interaction process found for the EAPC and the XYL system. In the latter, only a very small fraction of solvents is dissolved in water, therefore solvent diffusion from water to the polymer, which represents the fast exchange in the model described, is negligible. Xylene and 1-PeOH have thus to migrate from the nanodroplets directly to the polymer coating, and this results in a slower process.

It is critical to understand whether 1-pentanol is important because of its co-surfactant action, and thus its role is mediated by the structural features that it induces, or else if it plays the role of a co-solvent, which helps EA, PC and xylene in Paraloid B72 swelling. To address this point, 1-PeOH was removed from both EAPC and XYL. If pentanol is removed from the EAPC system a single-phase, optically transparent nanostructured system is obtained (EAPC-noPeOH). In contrast if this solvent is removed from XYL, no microemulsion is obtained. This observation again nicely illustrates the differences between EAPC (where the

Table 2 Composition of nanofluids investigated. In the table concentrations are reported as w/w \%. EAPC is the system presented in Section 2.7. $\mathrm{XYL}$ is the microemulsion described in the previous section. XYLTW is a microemulsion obtained by substituting the SDS/1-PeOH couple of the XYL system with Tween20, a nonionic surfactant, to obtain a monophasic system

\begin{tabular}{lccrc}
\hline & EAPC & EAPC-noPeOH & XYL & XYL-TW \\
\hline Water & 73.3 & 78.8 & 87.1 & 85.7 \\
SDS & 3.7 & 4 & 3.9 & \\
1-PeOH & 7 & & 6.5 & \\
PC & 8 & 8.6 & & \\
EA & 8 & 8.6 & 2.5 & 2.5 \\
Xylene & & & & 11.8 \\
Tween20 & & & & \\
\hline
\end{tabular}

continuous phase is mainly constituted by a mixture of water, propylene carbonate and ethyl acetate) and XYL. The role of surfactant and co-surfactant obviously is basic to maintain in a single aqueous phase an apolar solvent, like xylene.

XYL-TW (see Table 2) very slowly interacts with Paraloid B72 and when the system equilibrates small round droplets of partially swollen polymer remain stuck to the bottom and to the side of the glass test tube. Also in the case of EAPC-noPeOH the swollen polymer droplets are too dense to spontaneously detach from the glass of the test tube and remain stuck the bottom. This is not particularly surprising since $1-\mathrm{PeOH}$ is the less dense solvent in the EAPC formulation. Differently from the XYL-TW system, the interaction process of EAPC-noPeOH with Paraloid B72 is quite fast. Not as fast as the complete EAPC system, but the absence of 1-PeOH does not drastically slow down the interaction process.

We conclude that $1-\mathrm{PeOH}$ in the XYL system has a double role, it acts both as a structure builder of the system and it enhances the removal process of Paraloid B72, since it is quite abundant in the upper phase, which does not even form if this solvent is removed from the formulation. In the EAPC system it plays an important role as a co-solvent, while it seems that its cosurfactant nature is less important.

\subsection{Schematizing the interaction mechanism}

The interaction process for EAPC and XYL now can be schematized as follows (see Fig. 9). EAPC interacts in three distinct phases:

1. Solvents dissolved in the continuous aqueous phase, in equilibrium with dispersed droplets stabilized by a surfactant/ cosurfactant film, quickly interact with the polymer coating.
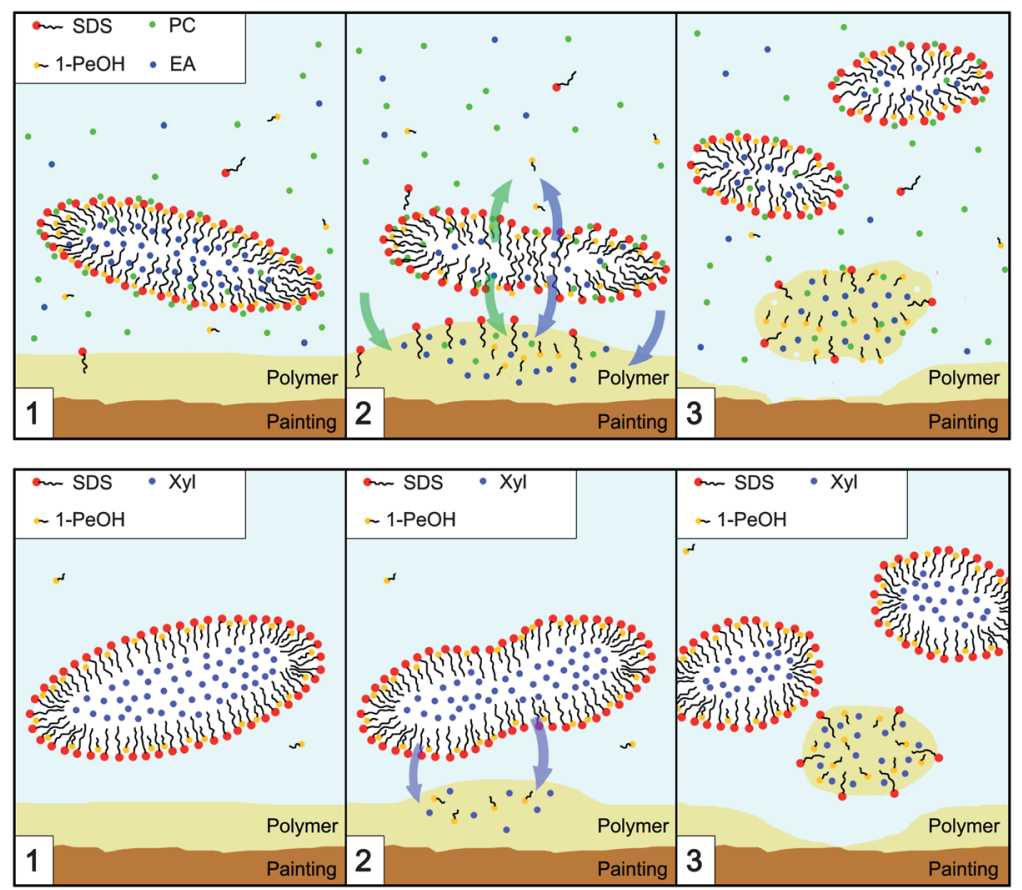

Fig. 9 Schematic representation of the interaction mechanism between the detergent nanostructured systems (top: EAPC; bottom: XYL) and the polymer coating. The top image is adapted from ref. 43 . 
2. A series of exchanges occurs, where solvents migrate from the aqueous phase to the polymer, from the nanodroplets (that act as dynamic nanocontainers) to the aqueous phase and from the nanodroplets to the polymer.

3. The polymer film "selects" an optimal composition that leads to swelling and chain disentanglement and detaches from the substrate, that now is clean, while the nanodroplets get smaller and re-organize their structure due to the outflow of the solvents.

It is worth pointing out some important features of this picture. First of all, the role of the nanodroplets is crucial; even if they do not seem to play a particularly direct role in the cleaning process, they make it possible that the right amount of organic solvents is dispersed in water in a single phase, and thus available to the polymer. The mixture of solvents absorbed by the polymer for the swelling process is selected by the polymer itself, according to its physical-chemical characteristics. For this reason it is clear that a cleaning system should be able to provide the polymer with the optimal solvent blend to perform a good removal.

A similar mechanism has been hypothesized for the XYL system in which the main difference resides in the fact that first step is missing; the process can be schematized as:

1. To reach the equilibrium, solvents migrate from the nanodroplets to the polymer. Actually a small fraction of $1-\mathrm{PeOH}$ is dissolved in the aqueous continuous phase, but its contribution is probably negligible.

2. The polymer extracts the optimal composition that leads to its swelling and detaches from the substrate, that now is clean, while the nanodroplets get smaller and re-organize their structure due to the outflow of the solvents.

We believe that a deep comprehension of all these factors is the key of a more conscious approach to the formulation of specifically tailored systems to face up with new conservative challenges. In this paper we presented a commentary about the results of a study that represents the first, yet very promising step towards this comprehension.

\subsection{Future steps}

Future works should be related to the study of the thermodynamic principles that rule the interaction process. In particular, since it seems that the polymer itself "chooses" the solvent blend to interact with, a study about polymer solubility in various pure solvents and solvent mixtures has already been undertaken in order to investigate this subject. Moreover a definite interest resides in reaching a predictive knowledge of the effectiveness of a detergent system before testing it, based on its physico-chemical properties. To achieve this ability we have to clearly identify the key factors that determine the efficacy of a polymer removal process. According to the results reported in this paper some of the most interesting features surely are the nanodroplets interfacial flexibility, solvency power of the organic components of the system and the possibility to have them partially dissolved in water, which could help in increasing the cleaning rate. A full understanding of these complex mechanisms will open new possibilities for a scientific approach to conservation of cultural heritage.

\section{Acknowledgements}

CSGI and MIUR are acknowledged for financial support. The access to the LLB neutron facility has been supported by the European Commission under the $7^{\text {th }}$ Framework Program: Integrated Infrastructure Initiative for Neutron Scattering and Muon Spectroscopy: NMI3/FP7 — Contract no. 226507. This work has been also partly realized with the financial support of TemArt; Programma Operativo Regionale-Regione Toscana, cofinanced by Fondo Europeo di Sviluppo Regionale (POR CreO FESR 2007-2013). Thanks are also due to Claudia Garcia Solis, conservator of the "Proyecto Arqueologico Mayapan" from INAH-Yucatan (Istituto Nacional de Antropologia e Historia; Mexico), Lilia Rivero Weber, Maria Del Carmen Castro and Yareli Jaidar Benavides, conservators from CNCPC (Coordinación Nacional de Conservación del Patrimonio Cultural), Mexico, for experiments in situ. Finally, thanks are due to INAH-CNCPC-CINAHY for their kind permission to reproduce the pictures about the archeological site of Mayapan.

\section{Notes and references}

1 R. Wolbers, Cleaning Painted Surfaces, Antique Collectors Club, Ltd, 2000.

2 P. Cremonesi, L'uso di Tensioattivi e Chelanti Nella Pulitura di Opere Policrome, Il Prato, Padova, 2001.

3 J. Estoe, Surfactant Aggregation and Adsorption at Interfaces, in Colloid Science, ed. T. Cosgrove, Blackwell Publishing Ltd, 2005.

$4 \mathrm{~K}$. Holmberg, B. Jonsson, B. Kronberg and B. Lindman, Surfactants and Polymers in Aqueous Solutions, Wiley, New York, 2004.

5 P. Mukerjee, C. Ramachandran and R. A. Pyter, J. Phys. Chem., 1982, 86, 3189-3197.

6 K. H. Raney, W. J. Benton and C. A. Miller, J. Colloid Interface Sci, 1987, 117, 282-290.

7 K. H. Raney and H. L. Benson, J. Am. Oil Chem. Soc., 1990, 67, 722 729.

8 K. H. Raney, J. Am. Oil Chem. Soc., 1991, 68, 525-531.

9 K. P. Bidyut and P. M. Satya, Curr. Sci., 2001, 80, 990-1001.

10 C. Tongcumpou, E. J. Acosta, L. B. Quencer, A. F. Joseph, J. F. Scamehorn, D. A. Sabatini, S. Chavadej and N. Yanumet, $J$. Surfactants Deterg., 2003, 6, 191-203.

11 C. Tongcumpou, E. J. Acosta, L. B. Quencer, A. F. Joseph, J. F. Scamehorn, D. A. Sabatini, S. Chavadej and N. Yanumet, J. Surfactants Deterg., 2003, 6, 205-213.

12 C. Tongcumpou, E. J. Acosta, L. B. Quencer, A. F. Joseph, J. F. Scamehorn, D. A. Sabatini, S. Chavadej and N. Yanumet, $J$. Surfactants Deterg., 2005, 8, 147-156.

13 D. Langevin, in Reverse Micelles, ed. P. Luisi and B. E. Straub, Plenum, New York, 1984, pp. 287-303.

$14 \mathrm{~J}$. Estoe, Microemulsions, in Colloid Science, ed. T. Cosgrove, Blackwell Publishing Ltd, 2005.

15 S. Friberg, in Microemulsion. Theory and Practice, ed. L. M. Prince, Academic Press, New York, 1970.

16 Industrial Application of Microemulsions, ed. C. Solans and H. Kunieda, Marcel Dekker, Inc., New York, 1997.

17 I. Danielsson and B. Lindman, Colloids Surf., 1981, 3, 391-392.

18 J. D. Mitchell and B. W. Ninham, J. Chem. Soc., Faraday Trans. 2, 1981, 77, 601-629.

19 J. N. Israelachvili, D. J. Mitchell and B. W. Ninham, J. Chem. Soc., Faraday Trans. 2, 1976, 72, 1525-1568.

20 W. Von Rybinski, Applications of Emulsions, in Modern Aspects of Emulsion Science, The Royal Society of Chemistry, Cambridge, 1998.

21 C. Stubenrauch, Microemulsions-Background, New Concepts, Applications, Perspectives, Wiley-Blackwell, 2008.

22 C. Solans, J. J. Garcia-Dominguez and S. Friberg, J. Dispersion Sci. Technol., 1984, 6(5), 523-537.

23 P. Erra, N. Azemar, M. R. Julia and C. Solans, J. Dispersion Sci. Technol., 1992, 13(1), 1-12. 
24 N. Azemar, J. Cot, P. Erra and C. Solans, in Compendium of Advanced Topics on Leather Technology, ed. J. Cot, Barcelona, 1991, vol. 1.

25 L. Borgioli, G. Caminati and G. Gabrielli, Sci. Tech. Cult. Herit., $1995,4,67-74$

26 E. Ferroni, G. Gabrielli and G. Caminati, Asportazione di Materiali Idrofobi da Superfici Pittoriche Murali Mediante Solubilizzazione in Sistemi Dispersi,in La Cappella Brancacci, la scienza per Masaccio, Masolino e Filippino Lippi, ed. Olivetti, Quaderni del restauro, 1992.

27 P. G. De Gennes and C. Taupin, J. Phys. Chem., 1982, 86, 2294-2304.

28 P. Mora, L. Mora and P. Philippot, Conservation of Wall Paintings, Butterworths, London, 1984.

29 E. De Witte, in Proceedings of the Symposium on Resins in Conservation, Scottish Society for Conservation and Restoration, Edinburgh, 1982.

30 R. L. Feller, Accelerated Aging: Photochemical and Thermal Aspects, The Getty Conservation Institute, Los Angeles, 1995.

31 L. Dei, E. Carretti and P. Baglioni, Proceedings of the 5th World Surfactants Congress-Cesio 2000, Florence, 2000, May 29-June 3, pp. $517-524$.

32 E. Carretti, L. Dei, C. Miliani and P. Baglioni, Prog. Colloid Polym. Sci., 2001, 118, 63-67.

33 C. Alba-Simionesco, J. Teixeira and C. A. Angell, J. Chem. Phys, 1989, 91, 395.

34 G. Rance and S. Friberg, J. Colloid Interface Sci., 1977, 60, 207.

35 P. Baglioni, L. Dei and E. Carretti, Italian Pat., FI/99/A/000071, 2 April 1999.

36 M. Baglioni, D. Berti, J. Teixeira, R. Giorgi and P. Baglioni, Langmuir, submitted.

37 E. Carretti, L. Dei and P. Baglioni, Langmuir, 2003, 19, 7867-7872.

38 C. V. Horie, Materials for Conservation, Butterworth, London, 1987

39 G. Palazzo, D. Fiorentino, G. Colafemmina, A. Ceglie, E. Carretti, L. Dei and P. Baglioni, Langmuir, 2005, 21, 6717-6725.

40 G. Colafemmina, D. Fiorentino, A. Ceglie, E. Carretti, E. Fratini, L. Dei, P. Baglioni and G. Palazzo, J. Phys. Chem. B, 2007, 111, 7184-7193.

41 S. Grassi, E. Carretti, P. Pecorelli, F. Iacopini, P. Baglioni and L. Dei, J. Cult. Heritage, 2007, 8, 119-125.

42 R. Giorgi, M. Baglioni, D. Berti and P. Baglioni, Acc. Chem. Res., 2010, 43, 695-704.

43 M. Baglioni, D. Rengstl, D. Berti, M. Bonini, R. Giorgi and P. Baglioni, Nanoscale, 2010, 2(9), 1723-1732.

44 D. Nickel, C. Nitsch, P. Kurzendorfer and W. Rybinsky, Prog. Colloid Polym. Sci., 1992, 89(1), 249-252.

45 K. Bobe and F. Tietze, Fett/Lipid, 1998, 100(2), 36-41.

46 H. Luders, Synthesis of Alkyl Glucosides and Alkyl Polyglucosides, in Nonionic Surfactants, Alkyl Polyglucosides, ed. D. Balzer and H. Luders, Marcel Dekker, New York, 2000, pp. 7-76.

47 E. Carretti, R. Giorgi, D. Berti and P. Baglioni, Langmuir, 2007, 23, 6396-6403.

48 R. Giorgi, D. Chelazzi, E. Carretti, E. Falletta and P. Baglioni, Proceedings of the 15th Triennal Conference ICOM Committee for Conservation, New Delhi, 2008, September 22-26, vol. I, pp. 527-533.

49 E. Ferroni, V. Malaguzzi-Valerj and G. Rovida, Proceedings of the ICOM Conference, Amsterdam, 1969.

50 E. Ferroni and P. Baglioni, Proceedings of the Symposium "Scientific Methodologies Applied to Works of Art”, Florence, 1984.

51 E. Ferroni, OPD Restauro, 1999, 11, 97.

52 M. Gridley, A Survey of Contemporary Material and Methods for Varnishing Removal, Final Year Project in Conservation, Courtald Institute of Art, London, 1991.

53 L. Goldberg, J. Am. Inst. Conserv., 1989, 28, 19-29.

54 N. Khandekar, Research into Potential Problems Arising from the Use of Aqueous Cleaning Systems, in Solvent Gels for the Cleaning of Works of Art: the Residue Question, ed. D. Stulik, D. Miller, N. Khanjian, R. C. Wolbers, J. Carlson, W. C. Petersen and V. Dorge,
Getty Trust Publications: Getty Conservation Institute, 2004, pp. 5$11 ; 12-17$.

55 R. C. Wolbers, in Preprints to the Conference Restoration '92: Conservation, Training, Materials and Techniques, Latest Developments, ed. V. Todd, J. Marsden, M. K. Talley, J. Lodewijks, V. Sluyterman and W. Koeno, IIC, London, 1992, pp. 74-75.

56 P. Cremonesi, A. Curti, L. Fallarini and S. Raio, Progetto Restauro, 2000, 15, 25-33.

57 R. C. Wolbers, Notes for Workshop on New Methods in the Cleaning of Paintings, Getty Conservation Institute, Marina del Rey, CA, 1990.

58 R. C. Wolbers, Conservation-Restauration des Biens Culturels, 1992, 4, 22-29.

59 A. Aggeli, M. Bell, N. Boden, J. N. Keen, P. F. Knowels, T. C. Mcleish, M. Pitkeathly and S. E. Radford, Nature, 1997, 386, 259-262.

60 M. Yoshida, M. Asano, T. Suwa and R. Katakai, Radiat. Phys. Chem., 1999, 55, 677-680.

61 S. Matsumoto, S. Yamaguchi, S. Ueno, H. Komatsu, M. Ikeda, K. Ishizuka, Y. Iko, K. Tabata, H. Aoki, S. Ito, H. Noji and O. Hamachi, Chem.-Eur. J., 2008, 14, 3977-3986.

$62 \mathrm{~K}$. Juntanon, S. Niamalang, R. Ruijiravanit and A. Sirivat, Int. J. Pharm., 2008, 356, 1-11.

63 E. Carretti, A. Macherelli, L. Dei and R. G. Weiss, Langmuir, 2004, 20, 8414-8418.

64 E. Carretti, L. Dei, R. G. Weiss and P. Baglioni, J. Cult. Heritage, 2008, 9, 386-393.

65 M. Bonini, S. Lenz, R. Giorgi and P. Baglioni, Langmuir, 2007, 23, $8681-8685$

66 M. Bonini, S. Lenz, E. Falletta, F. Ridi, E. Carretti, E. Fratini, A. Wiedenmann and P. Baglioni, Langmuir, 2008, 24, 1264412650.

67 E. Carretti, S. Grassi, M. Cossalter, I. Natali, G. Caminati, R. G. Weiss, P. Baglioni and L. Dei, Langmuir, 2009, 25, 8656-8662.

68 E. Carretti, I. Natali, C. Matarrese, P. Bracco, R. G. Weiss, P. Baglioni, A. Salvini and L. Dei, J. Cult. Heritage, 2010, 11, 373380.

69 P. Baglioni, L. Dei, E. Carretti and R. Giorgi, Langmuir, 2009, 25(15), 8373-8374

70 E. Carretti, E. Fratini, D. Berti, L. Dei and P. Baglioni, Angew. Chem., Int. Ed., 2009, 48, 8966-8969.

71 A. Chevalier, D. Chelazzi, P. Baglioni, R. Giorgi, E. Carretti, M. Stuke, M. Menu and R. Duchamp, Proceedings of the 15th Triennal Conference ICOM Committee for Conservation, New Delhi, September 22-26, 2008, vol. I, pp. 581-589.

72 A. Brizard, M. Stuart, K. Van Bommel, A. Friggeri, M. De Jong and J. Van Esch, Angew. Chem., Int. Ed., 2008, 47, 2063.

73 C. Stubenrauch, R. Tessendorf, R. Strey, I. Lynch and K. A. Dawson, Langmuir, 2007, 23, 7730.

74 C. Stubenrauch, R. Tessendorf, A. Salvati, D. Topgaard, T. Sottmann, R. Strey and I. Lynch, Langmuir, 2008, 24, 8473.

75 M. Magno, R. Tessendorf, B. Medronho, M. G. Miguel and C. Stubenrauch, Soft Matter, 2009, 5, 4763.

76 M. Muller, B. Stuhn, K. Busse and J. Kressler, J. Colloid Interface Sci., 2009, 335, 228-233.

77 A. Holmberg, P. Hansson, L. Piculell and P. Linse, J. Phys. Chem. B, 1999, 103, 10807-10815.

78 A. Holmberg, L. Piculell, P. Schurtenberger and U. Olsson, Colloids Surf., A, 2004, 250, 325-336.

79 K. J. Mutch, J. S. van Duijneveldt, J. Estoe, I. Grillo and R. K. Heenan, Langmuir, 2008, 24, 3053-3060.

80 K. J. Mutch, J. S. van Duijneveldt and J. Estoe, Soft Matter, 2007, 3, $155-167$.

81 C. Y. Hui and L. C. Wu, J. Appl. Phys., 1987, 61(11), 5129-5136.

82 D. F. Stamatialis, M. Sanopoulou and J. H. Petropoulos, Macromolecules, 2002, 35, 1021-1027. 